\title{
Blepharospasm followed by Parkinson's disease in two brother's case presentation in a family
}

\begin{abstract}
We report the history of two brothers who had early onset Parkinson (EOP) with blepharospasm, with family a history of Parkinson's disease. In both the neuropsychological examination showed cognitive deterioration with frontal lobe dysfunction. Patients with PLA2G6 mutation could show heterogeneous phenotype such as dystonia-parkinsonism (dystonia at onset affecting 14-57\% of EOP patients and off period dystonia as affecting 30-59\%), dementia and frontotemporal atrophy.

Conclusion: In some genetic types of PD is produced from the beginning a degeneration of multiple systems with deficit in multiple neurotransmitters.

Keywords: Parkinson's disease, blepharospasm, dystonia, genetic, familial, PARK14, healthy history, tremoric syndrome, clinical features, right upper limb, disorders, family cases, male patients, bromocriptine, genetic anticipation
\end{abstract}

Volume 2 Issue 4 - 2018

\author{
Julio López Argüelles,' Leydi María Sosa \\ Aguila, ${ }^{2}$ Aleima Rodríguez Carbajal, ${ }^{3}$ \\ Georgina Gonzalez Alba,' Lisan Montalvo \\ Manso' \\ 'Department of Neurology, University hospital Gustavo \\ Aldereguía Lima, Cuba \\ ${ }^{2}$ Department of Genetic, University hospital Paquito Gonzalez \\ Cueto, Cuba \\ ${ }^{3}$ Department of Neuropsicology, University hospital Gustavo \\ Aldereguía Lima, Cuba
}

\begin{abstract}
Correspondence: Julio López Argüelles, Department of Neurology, University hospital Gustavo Aldereguía Lima, Cienfuegos, Cuba,

Email julito.arguelles@gmail.com, juliola@jagua.cfg.sld.cu
\end{abstract}

Received: June 06, 2018| Published: August 30, 2018

\section{Introduction}

Parkinson's disease (PD) was described for first time in 1817 by James Parkinson. In 1888 Gowers introduces the possibility of a hereditary disease. Subsequently the studies of cases-controls have made it possible to identify a history of Parkinson family between $13 \%$ and $33 \%$ of the cases, ${ }^{1-8}$ what presumes that the relatives of patients with PD have a risk between 3.5 and 14.6 times greater than the relatives of the controls in order to develop the disease. ${ }^{2}$ The heredity pattern of many of these cases is compatible with a dominant autosomal heredity with reduced penetrance, being the maximum risk of developing the disease of $20 \%$ for the relatives of first degree. ${ }^{8}$ Some studies have in addition demonstrated the existence of a genetic anticipation; however, have not been able to be identified differentiating clinical features among the family cases and the sporadic in the majority of studies, except for a trend an earlier beginning of the disease in the family cases in one of these studies. ${ }^{3}$ Some types of PD with a demonstrated genetic pattern have a beginning which is early and/or associated with other movement disorders, basically dystonia. ${ }^{6}$ Our objective is to show the case of two brothers had an early onset Parkinson's disease as blepharospasm.

\section{Case presentation}

We report the history of two male patients, brothers who had early onset Parkinson Disease (EOP) with blepharospasm and tremor of the right arm, with family history of Parkinson's disease: father and mother, which initiated and maintained their evolution with predominance of tremor. The younger brother (YB) is 55 years old, rural origin, healthy history, begins at 47 years old with involuntary movements in both eyes (blepharospasm) and approximately 8 months later begins with tremor in the right upper limb, followed by slow installation and progressively of a light rigid-acinétic syndrome that after 2 years of evolution was already bilateral. Begin treatment with dopaminérgic agonist (bromocriptine) and amantadine the first 2 years of the treatment, then started to treat with levodopa/carbidopa, with an adequate response, currently remaining in a stage 3 of the disease and UPDRSmotor $=28$. The older brother $(\mathrm{OB})$ with 59 years old, of urban origin, begins also with blepharospasm at 54 years old followed by a tremoric syndrome of the right arm, with a slower evolution than his brother and a better response to the treatment with bromocriptine and amantadine (UPDRSmotor=14). Both brothers after initiated the treatment began with great improvement of blefarospasm and Parkinsonian symptoms. In both the neuropsychological examination showed cognitive deterioration with frontal lobe dysfunction (Montreal Cognitive Assessment: $\mathrm{YB}=16 ; \mathrm{OB}=17$, Fontal Assessment Battery: $\mathrm{YB}=10 ; \mathrm{OB}=13$ ), furthermore began with sleep disorders a year before the motor symptoms. We ordered studies in order to rule out Parkinson plus: ceruloplasmin in serum and urine were normal, liver study without alteration, neuro-ophthalmological study rule out the presence of Keyser-Fletcher's rings, the MRI showed light cortical atrophy. Until now they have not appeared patient sick in the IV generation although these do not exceed still the 40 years old.

\section{Discussion}

The majority of PD cases are sporadic and only about $10 \%$ of patients report a positive family history. ${ }^{9}$ Out of the six genes unequivocally linked to heritable, monogenic PD, mutations in SNCA (PARK 1-4), and LRRK2 (PARK8) are responsible for autosomal dominant PD forms, and mutations in Parkin (PARK2), PINK1 (PARK6), DJ-1 (PARK7), and ATP13A2 (PARK9) are accountable for PD that displays an autosomal recessive (AR) mode of inheritance. ${ }^{6}$ Apart from the genes causing the six monogenic forms of PD, changes in a large number of additional genes were considered PD-causative: UCHL1 (PARK5), GYGYF2 (PARK11), OMI/HTRA2 (PARK13), PLA2G6 (PARK14), and FBXO7 (PARK15). ${ }^{6}$ Mutations of PLA2G6 gene have been lately proposed to be the causative gene for PARK14 in patients with autosomal recessive young-onset parkinsonism (YOPD). ${ }^{7}$ Patients with PLA2G6 mutation could show heterogeneous 
phenotype such as dystonia-parkinsonism (dystonia at onset affecting $14-57 \%$ of YOPD patients and off period dystonia as affecting $30-$ $59 \%),{ }^{5-10}$ dementia, frontotemporal atrophy/hypoperfusion, with or without brain iron accumulation. Based on the clinical heterogeneity, the functional roles of PLA2G6 and the roles of PLA2G6 variants including single heterozygous mutations should be further elucidated in patients with atypical parkinsonism, dementia, or PD. ${ }^{11}$ In addition rapid-onset dystonia-parkinsonism (RDP) (also known as DYT12) is characterized by the abrupt onset of dystonia and parkinsonism and is caused by mutations in the ATP $1 A 3$ gene. But the phenotype includes abrupt onset of dystonia with features of parkinsonism, a rostrocaudal gradient, and prominent bulbar findings. Other features are minimal or no tremor at onset, occasional mild limb dystonia before the primary onset, lack of response to dopaminergic medications, rare abrupt worsening of symptoms later in life, stabilization of symptoms within a month and minimal improvement overall (Figure1). ${ }^{11,12}$

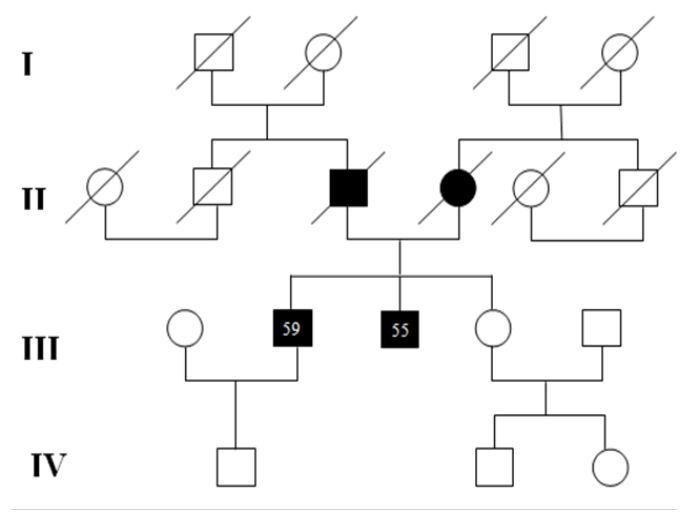

Figure I Pedigrees of the brother family, showing the onset Parkinson's disease in second generation. Black symbols - affected individuals; white symbols - unaffected individuals.

\section{Conclusion}

The previous case shows the phenotypical heterogeneity of onset of the PD, with great variability of symptoms mainly blepharospasm, dementia with dysfunction of the frontal lobe and sleep alteration, which it demonstrates that in some genetic types of PD is produced from the beginning a degeneration of multiple systems with deficit in multiple neurotransmitters.

\section{Acknowledgements}

None.

\section{Conflict of interest}

The author declares there is no conflict of interest.

\section{References}

1. Anheim M, Elbaz A, Lesage S, et al. Penetrance of Parkinson disease in glucocerebrosidase gene mutation carriers. Neurology. 2012;78(6):417420.

2. Autere JM, Moilanen JS, Myllyla VV, et al. Familial aggregation of Parkinson's disease in a Finnish population. J Neurol Neurosurg Psychiatry. 2000;69:107-109.

3. Bonifati V, Fabrizio E, Vanacore N, et al. Familial Parkinson's disease: a clinical genetic analysis. Can J Neurol Sci. 1995;22(4):272-279.

4. De MG, Filla A, Marconi R, et al. A genetic study of Parkinson's disease. J Neural Transm Suppl. 1995;45:21-25.

5. Kidron D, Melamed E. Forms of dystonia in patients with Parkinson's disease. Neurology. 1987;37:1009-1011.

6. Klein C, Westenberger A. Genetics of Parkinson's disease. Cold Spring Harb Perspect Med. 2012;2(1):a008888.

7. Lu CS, Lai SC, Wu RM, et al. PLA2G6 mutations in PARK14-linked young-onset parkinsonism and sporadic Parkinson's disease. Am J Med Genet B Neuropsychiatr Genet. 2012;159B(2):183-191.

8. Paul KC, Schulz J, Bronstein JM, et al. Association of Polygenic Risk Score with Cognitive Decline and Motor Progression in Parkinson Disease. JAMA Neurol. 2018;75(3):360-366.

9. Thomas B, Beal MF. Parkinson's disease. Hum Mol Genet. 2007;16 Spec No. 2:R183-R194.

10. Wickremaratchi MM, Knipe MD, Sastry BS, et al. The motor phenotype of Parkinson's disease in relation to age at onset. Mov Disord. 2011;26(3):457-463.

11. Yoshino H, Tomiyama H, Tachibana N, et al. Phenotypic spectrum of patients with PLA2G6 mutation and PARK14-linked parkinsonism. Neurology. 2010;75(15):1356-1361.

12. Zaremba J. Rapid-onset dystonia-parkinsonism. Neurology. 2005;62(12):1296-1297. 